Recepción: 15 / 04 / 2017

Aceptación: 01 / 05 / 2017

Ciencias Agrícolas

Publicación: 15 / 05 / 2017

Articulo Científico

\title{
Control biológico de mosca blanca (Aleurothrixus floccosus maskell) mediante chrysoperla carnea en el cultivo de banano
}

Biological control of whitefly (Aleurothrixus floccosus maskell) by chrysoperla carnea in banana cultivation

\section{Controle biológico de moscas brancas (Aleurothrixus floccosus Maskell) por carnea Chrysoperla no cultivo de banana}

\author{
Rafael V. Pluas-Pilozo \\ rpluas@uagraria.edu.ec \\ Alexandra A. Navarrete-Cornejo" \\ anavarrete@uagraria.edu.ec \\ Nuvia L. Morán-Sanchez ${ }^{\text {"m }}$ \\ nmoran@uagraria.edu.ec
}

Correspondencia: rpluas@uagraria.edu.ec

\footnotetext{
Magister en Agroecología y Agricultura Sostenible; Ingeniero Agrónomo; Tecnólogo en Banano y Frutas Tropicales; Universidad Agraria del Ecuador, Ecuador.

II. Magister en Agroecología y Agricultura Sostenible; Diploma Superior en Diseño Curricular por Competencias; Ingeniero Agrónomo; Universidad Agraria del Ecuador, Ecuador.

III. Magister en Agroecología y Agricultura Sostenible; Ingeniera Agrónoma; Universidad Agraria del Ecuador, Ecuador.
} 


\section{Resumen}

La presente investigación da a conocer la importancia que ha tenido el control biológico en uno de los cultivo de mayor importancia en nuestro país como es el banano que nos permite obtener mayor ingreso económico. Esta investigación será el mejor medio para difundir esta información a través de una serie de labores agrotecnia para un control de la mosca blanca A. floccosus no es una solución a la radicación total del insecto pero su estudio determinado que es una técnica para reducir el uso de los agroquímicos. Este estudio significa sin duda alguna, dar un paso al cambio, la metodología de la investigación es de proyecto factible debido o que se trata de una propuesta para resolver un problema. Se obtuvo resultados muy favorables ya que la propuesta se realizó en el tiempo establecido. La investigación difunde la importancia del control biológico y el rico potencial visual que transmite, de una forma clara, precisa y de fácil manejo. Con este nuevo aporte se enriquecerá sin duda alguna las fuentes hidrográficas, principalmente en la biblioteca de la "Universidad Agraria del Ecuador”; Facultad de Ciencias Agrarias.

Palabras clave: Control biológico Chrysoperla carnea; mosca blanca A. floccosus; cultivo de banano. 


\begin{abstract}
This research discloses the significant role played by biological control in one of the most important crop in our country like bananas that allows us to get more income. This research is the best way to disseminate this information through a series of agrotecnia work to control whitefly A. floccosus is not a solution to the total filing bug but his particular study is a technique to reduce the use of agrochemicals. This study means undoubtedly step change, the research methodology is feasible project due or that it is a proposal to resolve a problem. Very favorable results were obtained since the proposal was made in the set time. Research disseminates the importance of biological control and rich visual potential that transmits a clear, precise and easy to use. With this new contribution will certainly enrich river sources, mainly in the library of the "Agrarian University of Ecuador"; Faculty of Agricultural Sciences.
\end{abstract}

Key words: Biological control Chrysoperla carnea; whitefly A. floccosus; banana cultivation. 


\section{Resumo}

Esta pesquisa revela o papel significativo desempenhado pelo controle biológico em uma das culturas mais importantes em nosso país como as bananas que nos permite obter mais renda. Esta pesquisa é a melhor maneira de disseminar esta informação através de uma série de agrotecnia trabalho para controlar a mosca-branca A. floccosus não é uma solução para o bug de arquivamento total, mas seu estudo particular é uma técnica para reduzir o uso de agroquímicos. Este estudo significa, sem dúvida, mudança de etapa, a metodologia de pesquisa é viável projeto devido ou que é uma proposta para resolver um problema. Obtiveram-se resultados muito favoráveis desde que a proposta foi feita no tempo estabelecido. A pesquisa divulga a importância do controle biológico e rico potencial visual que transmite uma clara, precisa e fácil de usar. Com esta nova contribuição certamente enriquecerá as fontes de rios, principalmente na biblioteca da "Universidade Agrária do Equador"; Faculdade de Ciências Agrárias.

Palavras chave: Controlo biológico Chrysoperla carnea; Mosca branca A. floccosus; Banana. 


\section{Introducción.}

A la actualidad se estima que en el Ecuador existen 230.000 hectáreas sembradas de banano, de las cuales el $68.30 \%$ están tecnificadas, el $21.2 \%$ semitecnificadas y un $10.5 \%$ no tecnificadas. Se encuentran distribuidas principalmente en la Provincia de El Oro con el $33.44 \%$; en el Guayas con el 30.20 \%; en Los Ríos el $28.44 \%$; en el Cañar $3.50 \%$ y Esmeraldas $2.81 \%$. Esta superficie está repartida en 5.322 haciendas cuyos propietarios son 4.739 productores. El rendimiento promedio es de 35 TM / hectárea. (Giffoni, Valera, Díaz, \& Vásquez, 2007)

Desde 1 a 50 hectáreas representa el 36\% de la superficie sembrada y está en manos del 80\% de los productores; desde 51 a 100 hectáreas representa el 38\% de la superficie sembrada y está en manos del $17 \%$ de los productores y desde más de 100 hectáreas representa el $26 \%$ de la superficie sembrada y está en manos del $3 \%$ de los productores. (Silvia N, Riquelme, \& Botto, 2010)

Este cultivo de ciclo perenne permite obtener divisas que generan una considerable cantidad de trabajo, de bienes y servicios. En el país el banano presenta la mayor actividad agrícola, constituyéndose como el primer país exportador de fruta con 33'667.458 TM. (Toneladas métricas) en el año 2001. (Hoddel, Van Driesche, \& Sanderson, 1998)

En muchas ocasiones se ha llevado el control convencional (Químico) en varias haciendas bananeras dando como resultados la degradación del suelo y la contaminación del medio ambiente, la resistencia de insectos-plagas a los productos químicos y por ultimo afectando la vida humana.

La Agroecología a menudo incorpora ideas sobre un enfoque de la agricultura más ligado al medio ambiente y más sensible socialmente, se centra no solo en la producción sino también en la 
sostenibilidad ecológica del sistema de producción, en las relaciones ecológicas presentes en el campo y su propósito es iluminar la forma, la dinámica y las funciones de esta.

La Agroecología es una disciplina que nos da herramienta para el manejo de los recursos en los sistemas de producción. Pero su adopción es necesario considerar un proceso inicial de diagnóstico que permita caracterizar los problemas y potencialidades del sistema del sistema de producción; y una segunda fase donde el agricultor comenta sus necesidades básicas limitantes e innovaciones tecnológicas (Héctor, S. Altiere M. 1997).

El presente estudio busca controlar la mosca blanca A. floccosus mediante liberaciones de Ch. carnea en el cultivo de banano en la hacienda PRIMOBANANO en el cantón Marcelino Maridueña Provincia del Guayas.

\section{Materiales y métodos.}

\section{Aspecto metodológico}

En el presente trabajo investigativo se realizaron diferentes actividades para alcanzar los objetivos propuestos usando los materiales y métodos descritos a continuación.

\section{Ubicación}

El presente trabajo de investigación se realizó en la Hacienda Primobanano propiedad del Ing. Mariano Gonzales ubicado en el Rcto. Rio viejo cantón Marcelino Maridueña Provincia del Guayas, Km 8.5 vía a Puente Payo, cuyas coordenadas geográficas son latitud sur $02^{\circ}-15^{\prime}-00^{\prime \prime}$ y latitud oeste $79^{\circ}-28^{\prime}-30^{\prime \prime}$ con una altitud de $80 \mathrm{msnm} .{ }^{*} 1 /$ 
Los parámetros meteorológicos que influyen en la zona son; temperatura promedio anual es de $26^{\circ} \mathrm{C}$, humedad relativa $70 \%$, precipitación promedio anual es de $1529 \mathrm{~mm}$ y luminosidad de 806 horas luz/día.*2/

Los suelos en que se llevaron a cabo la investigación presenta una pendiente algo pronunciada, de textura franco - arcillosa, $\mathrm{pH}$ prácticamente neutro, bajo en materia orgánica.

*1/. Basado en los archivos del departamento fitosanitario.

*2/. Fuente, estación meteorológica de la finca Primobanano.

\section{Factores en estudio}

Los factores en estudio para este trabajo de la investigación científica fuero cuatro, tres con liberaciones de insectos depredadores y un testigo absoluto (sin liberar), el insecto benéfico que se libero fue Ch. carnea reproducido en laboratorio en un lapso de 5,6 meses para obtener un pie de cría y el número necesario para la investigación.

Este diseño estuvo compuesto de 4 tratamientos. En el cuadro $\mathrm{N}^{\circ} 1$ se detalla el número de Ch. carnea por tratamiento para el control de A. floccosus en el cultivo de banano.

\section{Análisis estadístico}

En la presente investigación, se planteó un diseño completamente al azar (DBCA). Los efectos fueron evaluados a través de 10 repeticiones (10 plantas) seleccionadas al azar; utilizando el análisis de varianza cuyo esquema se indican en el cuadro $\mathrm{N}^{\mathbf{2}}$ 2. La comparación de análisis de promedio se realizó mediante la prueba de TUKEY, al $5 \%$ de probabilidad. 


\begin{tabular}{|c|c|}
\hline $\begin{array}{c}\mathbf{N}^{\circ} \text { DE } \\
\text { TRATAMIENTO }\end{array}$ & NUMERO DE CHRYSOPAS POR TRATAMIENTO \\
\hline 1 & 100 huevos de chrysopa en plantas de 8 sem de edad \\
\hline 2 & 200 huevos de chrysopa en plantas de 8 sem de edad \\
\hline 3 & 300 huevos de chrysopa en plantas de 8 sem de edad \\
\hline 4 & TESTIGO ABSOLUTO \\
\hline
\end{tabular}

Análisis de varianza

\begin{tabular}{|c|c|}
\hline FUENTE DE VARIACION & $\begin{array}{c}\text { GRADOS DE } \\
\text { LIBERTAD }\end{array}$ \\
\hline TOTAL & 39 \\
\hline TRATAMIENTOS & 3 \\
\hline ERROR EXPERIMENTAL & 36 \\
\hline
\end{tabular}

Cuadro $N^{o}$ 2.- Tabla de análisis de varianza

Material vegetativo

Para llevar a cabo esta investigación se utilizó plántulas meristemáticas, certificadas procedente de la empresa Agripac (variedad, William) cuyas características son las siguientes:

Cada planta de banano tiene un promedio de 4 semanas de su ciclo de vida, con una emisión foliar de 5 a 7 días cada hoja.

Su altura tiene $0.70 \mathrm{mts}, 28 \mathrm{~cm}$ diámetro de fuste

Manejo del experimento

Para la implementación de los tratamientos se realizaron las siguientes actividades. 


\section{Preparación del terreno}

Para el efecto, antes de la realizar de la siembra se efectuó un pase de arado de disco a una profundidad de $30 \mathrm{~cm}$ y uno de rastra con el propósito de dejar aireado el suelo, la nivelación se efectuó con la ayuda del tractor de tal forma que se fueron eliminando las malas hierbas y desechos no deseados. Se instaló el sistema de riego sub-foliar con el distanciamiento correspondiente de $10 \mathrm{~m}$ x $11 \mathrm{~m}$, luego de esto se delimitaron los bloques y parcelas experimentales.

\section{Trazado de parcela}

Se efectúo la delimitación del terreno con estacas, cinta métrica y piola, cada parcela tuvo una medida de 30m de largo por 20m de ancho con un área total de $600 \mathrm{~m} 2$

\section{Siembra}

Antes de efectuar la siembra se seleccionó aquellas plantas que tuviesen características apropiadas es decir que no estén cloróticas, sin estropeo o con malformaciones en los entrenudos de cada base de la hoja (plantas mutantes).

Se procedió balizar y hacer huecos a una profundidad de $30 \mathrm{~cm}$ y un diámetro de $15 \mathrm{~cm}$ para cada unidad de producción.

Riego

Una vez culminado con la labor de siembra, se efectúo el riego vía sub-foliar en tiempo determinado de 3 horas hasta obtener capacidad de campo. El banano es un cultivo muy exigente en agua, por ende en plantas sembradas por primera vez, se efectúa el riego dos veces al día, por la 
mañana y en horas de la tarde en un tiempo determinado de dos hora diaria, dependiendo de las temperaturas que ocurre en el día.

\section{Control de malezas}

Cuando transcurrieron 4 a 5 semanas desde la siembra, aparecieron las primeras malezas de forma espontaneas, compitiendo con el cultivo la absorción de agua, nutrientes y minerales. Es aconsejable su eliminación por medio del control manual utilizando Machete, pala, azadón pero no utilizar agroquímicos en especial en las doce primeras semanas de edad.

\section{Fertilización}

La planta de banano necesita para su desarrollo los macro, micronutrientes y minerales. Los sistemas de estos elementos la planta se manifiesta cuando algún nutriente o mineral esta en defecto o en exceso.

Se recomienda una fertilización al suelo desde la siembra hasta culminar su ciclo biológico.

Las siembras por primera vez se realizan un programa de fertilización. En el cuadro $\mathrm{N}^{\mathrm{o}} 3$ se detalla lo indicado. 


\begin{tabular}{|c|c|c|c|c|c|c|c|c|c|c|}
\hline \multicolumn{11}{|c|}{ PROGRAMA DE FERTILIZACION PARA SIEMBRA" R.0, R1" } \\
\hline SEM & CALCIO & AMONIO & \begin{tabular}{|l} 
Mg. \\
Granu \\
lado
\end{tabular} & \begin{tabular}{|c|} 
Mg. \\
Estan \\
dar
\end{tabular} & $\begin{array}{c}\text { Muriato } \\
\text { (K) } \\
\text { Estandar }\end{array}$ & $\begin{array}{c}\text { Muriato } \\
\text { (K) } \\
\text { Granulado }\end{array}$ & UREA & DAP & CAUNTER & $\begin{array}{c}\text { Sulfato de } \\
\text { Potacio } \\
\text { Granulado }\end{array}$ \\
\hline 0 & 350 & & & & & 60 & & 80 & $7+7$ & \\
\hline 1 & 250 & 40 & & & & & & & & \\
\hline 2 & & 40 & & & & & & & & \\
\hline 3 & & 40 & & & & & & & & \\
\hline 4 & & 50 & & 60 & & & & & & \\
\hline 5 & & 50 & & & & & & & & \\
\hline 6 & & 50 & & & 40 & & & & & \\
\hline 7 & & 50 & & & & & & & & \\
\hline 8 & & 50 & & & 20 & & & & & \\
\hline 9 & & 50 & & & 20 & & & & & \\
\hline 10 & 400 & 50 & & & 20 & & & & & \\
\hline 11 & & 50 & & & 20 & & & & & \\
\hline 12 & & 90 & & & 40 & & & & & \\
\hline \multicolumn{11}{|l|}{13} \\
\hline 14 & & & 90 & & & 40 & 40 & & & \\
\hline 15 & 400 & & & & & & & & & \\
\hline 16 & & & & & & 40 & 40 & & & \\
\hline \multicolumn{11}{|l|}{17} \\
\hline 18 & & & & & & 40 & 40 & & & \\
\hline \multicolumn{11}{|l|}{19} \\
\hline 20 & & & 100 & & & 40 & 40 & & & \\
\hline \multicolumn{11}{|l|}{21} \\
\hline 22 & & & & & & 40 & 40 & & & \\
\hline \multicolumn{11}{|l|}{23} \\
\hline 24 & & & & & & 40 & 40 & & & \\
\hline \multicolumn{11}{|l|}{25} \\
\hline 26 & & & & & & 35 & 30 & & & \\
\hline \multicolumn{11}{|l|}{27} \\
\hline 28 & & & & & & 35 & 30 & & & \\
\hline 29 & 500 & & & & & & & & & \\
\hline 30 & & & & & & 35 & 30 & 50 & & \\
\hline 31 & & & 100 & & & & & & & \\
\hline 32 & & 40 & & & 35 & & & & & \\
\hline \multicolumn{11}{|l|}{33} \\
\hline 34 & & 40 & & & 35 & & & & & \\
\hline \multicolumn{11}{|l|}{35} \\
\hline 36 & & 60 & & & 35 & & & & & \\
\hline
\end{tabular}

Tabla $N^{o}$ 3.- Programa de fertilización en banano en mini plantías 


\section{Control de sigatoka}

Se llevó un control fitosanitario a partir de los 14 días en que la planta emita su primera hoja, es decir en la segunda semana de edad utilizando producto de contacto como son los mancoceb o clorotaloniles, mezclando con foliares ricos en minerales y nutrientes como macro y micronutrientes quelatados para su rápida asimilación. Esta mezcla se las aplica con bomba CP3 a una frecuencia de siete días hasta la semana diez, en donde se complica fumigar con este tipo de equipo, es cuando se la remplaza con bomba de motor hasta la semana quince, aislando la motobomba para darle pasó a las fumigaciones aéreas.

\section{El deshermane y la selección}

Se la realizo a partir de la ocho semana de edad, eliminando todo hermano profundo proveniente de la primera corona que está ligado en el fondo y centro del cormo de la planta, esta práctica se efectuó a una frecuencia de tres semana hasta la semana catorce, es cuando las yemas vegetativas de la segunda y tercera corona se comienza tomar forma para dar paso a una nueva unidad de producción.

\section{La floración y cosecha de fruto}

El promedio de la floración se dio a partir de la semana veinte y dos, con un número de ocho manos por racimo, ya hecha la poda de los dedos falsos por ende es aceptable para la producción.

La cosecha se la realizo a las treinta y dos semanas de edad. Se utilizó una herramienta conocida con en el nombre de guadaña que consiste en tener un machete cortado y con filos en ambos lados con una medida de treinta $\mathrm{cm}$ de largo por seis $\mathrm{cm}$ de ancho y adherida a una palanca y 
con la ayuda de una cuna para cargar la fruta hasta la línea donde va enganchada en una garrucha para llevarla a la empacadora y darle su respectivo proceso de empaque.

\section{Liberaciones de ch. Carnea en los tratamientos}

Esta liberación se realizó en la semana 21 después de la toma de datos en campo en la semana 20 donde se estableció la población general de $\boldsymbol{A}$. floccosus con sus estadios ninfales para proceder las liberaciones de $\boldsymbol{C h}$. carnea, en sus respectivas plantas escogidas al azar y en la hoja uno.

La Ch. carnea, se puede liberar en estado de larva como controlador, o en estado de adulto como colonizador. Pero esta investigación se enfocó en liberar estados de huevos en plaquetas o trozos de cartulina en donde los adultos ovipositaron sus posturas en los cilindros y fueron retirados recortados y contabilizados cada uno, para luego ser llevados al campo y liberar en los tratamientos correspondiente tal como se lo tubo provisto para su respectiva evaluación.

Teniendo listo el material procedemos a escoger 5 plantas al azar a una edad promedia de 14 semanas, se decidió hacer liberaciones a esa edad por motivo del riego, en esa altura no alcanza a mojar las placas donde están los huevos de las Ch. carnea.

Procedemos a retirar y recortar las cartulina procedente de los tambores o tubos cilíndrico donde los adultos ovipositaron sus posturas, las plaquetas van sujetado por un alambre en forma de gachos y adherido a la nervadura central a unos $40 \mathrm{~cm}$ del ápice de las hojas número 1 en donde se va a tomar los datos por semana en esa hoja durante su ciclo de vida útil correspondientes a partir del décimo día. 


\section{Frecuencia de evaluación}

Para determinar el comportamiento de Ch. carnea, en los tratamientos en donde se liberó en diferentes dosis, se procede a realizar evaluaciones a una frecuencia de siete a diez días respectivamente.

\section{Resultados.}

Reproducción masiva de ch. Carnea. En el laboratorio

Para la producción de $\boldsymbol{C h}$. carnea se han desarrollado técnicas para la multiplicación masiva como son:

\section{Unidad de crianza de Ch. carnea}

Para iniciar esta cría se recolectaron adultos del campo (en el cultivo de banano), con la ayuda de la manga entomológica, luego se colocaron en unos tubos cilíndricos de cartón. Estos recipientes son acondicionados con tapa de tela tul para darle ventilación; dentro de los baldes se coloca tubos hecho de cartulina en donde ovipositan los adultos, este método sirvió como para la reproducción, oviposición y para la obtención de huevos, posteriormente se retiró los huevos y se colocó en unas bandejas plásticas, en un lapso de 8 días estos eclosionaron y las larvas completaron su ciclo, convirtiéndose en pupas en un tiempo determinado emergen los adultos, este ciclo fue repetido por algunas ocasiones.

Para la crianza de las larva se utilizó bandejas plásticas y en la tapa se realizó una abertura de $5 \times 8 \mathrm{~cm}$ donde se colocó tela tul para darle ventilación y puedan respirar las larvas, en el interior de la bandeja se colocaron cartulinas dobladas a manera de acordeón para dividirlas y evitar el canibalismo entre ellas, como alimento de las larvas se utilizan huevos frescos de Sitotroga 
cerealella, la tapa de las bandejas están provistas de una tela tul para darle ventilación y puedan respirar las larvas.

Este insecto completa su ciclo larval en un promedio de 14 días, en cada bandeja se colocan hasta 80 larvas, la alimentación se realiza cada 2 días.

Una vez cumplido su desarrollo larval, a los días de haber instalado las unidades de crianza larval (Bandeja), conforme se van formando los cocones en las cartulinas, estas se retiran y se remplazan por otra, de esta manera evitamos que se dañen y puedan completar su desarrollo.

Los cocones son trasladados a una jaula con mayas metálicas en donde después de 12 días aproximadamente emergen los adultos, para luego de 2 días ser trasladados a la unidad de oviposición (tubos cilíndricos).

\section{Alimentación de los adultos}

Como alimentación para los adultos se utiliza una mezcla de 8 cucharadas de levadura de cerveza en polvo, 5 cucharadas de agua, 5 cucharadas de miel de abeja, 1/2 cucharada de polen, parte de esta mezcla se coloca sobre una placa rectangular de $2 \times 5 \mathrm{~cm}$. y la introducimos dentro de los tubos cilíndricos; así mismo se coloca algodón embebido en agua, la colocación del alimento se realiza diariamente. El alimento se la guarda en la nevera a una temperatura de 15 a $18^{\circ} \mathrm{C}$ para mantener su concentración y no se descomponga. 


\section{Cosecha de huevos}

Para cosechar los huevos de las unidades de oviposición es necesario efectuar un cambio de balde para trasladar a los adultos, una vez realizado el cambio se recolectan los huevos con la ayuda de un pincel, luego se cuentan y se los ubica en cajas petrix para ser conservados en congelación a una temperatura de $18^{\circ} \mathrm{C}$, por un lapso no más de 20 días, durante este período los huevos pueden ser utilizados como pie de cría o pueden ser liberados para ejercer un control al campo.

En la actualidad se está incrementando la cantidad de unidades de oviposición para en un futuro realizar liberaciones masivas de huevos. Para el control de huevos y ninfas de algunas plagas de importancia en el cultivo de caña de azúcar y en plantilla de banano para el control de ácaros y mosca blanca.

\section{Incidencia de mosca blanca}

\section{Datos tomados en campo}

Antes de realizar las liberaciones, se efectuó una primera evaluación con la finalidad de diagnosticar el número de poblaciones, escogiendo 10 plantas al azar en cada parcela y comparar el antes y el después, con las liberaciones de $\boldsymbol{C h}$. carnea en cada uno de los tratamientos.

\section{Evaluación de la mosca blanca en sus estados}

A continuación se detalla los promedios de las evaluaciones, en cada uno de los tratamientos en las parcelas experimentales, donde se observó los diferentes estados biológicos de A. floccosus 


\section{Muestreo en el tratamiento 1}

\begin{tabular}{|c|c|c|c|c|c|}
\hline \multirow{2}{*}{ Estados Biologicos } & \multicolumn{5}{|c|}{ Evaluación de mosca blanca en plantias en el tratamiento 1} \\
\hline & Hoja $N^{\circ} 1$ & Hoja N`2 & Hoja N`3 & Hoja $N^{\circ} 4$ & Promedio \\
\hline Adultos & 14 & 4 & 2 & 5 & 6,25 \\
\hline$N^{\circ}$ de Posturas & 17 & 14 & 16 & 8 & 13,75 \\
\hline Huevos & 18 & 10 & 3 & 0 & 7,75 \\
\hline Ninfas L1 & 16 & 18 & 0 & 0 & 8,5 \\
\hline Ninfas L2 & 12 & 16 & 7 & 7 & 10,5 \\
\hline Ninfas L3 & 0 & 0 & 9 & 11 & 5 \\
\hline Ninfas L4 & 0 & 0 & 10 & 9 & 4,75 \\
\hline Emisión Foliar & 0,70 & 0,70 & 0,70 & 0,70 & 0,70 \\
\hline Frecuencia & & & & & 7 Dias \\
\hline
\end{tabular}

En el cuadro se detalla las poblaciones promedios del tratamiento $\mathrm{N}^{\circ} 1$, la incidencia de mosca blanca $A$. floccosus en sus estados biológicos.

\section{Muestreo en el tratamiento 2}

En el cuadro se detalla las poblaciones promedios, del tratamiento $\mathrm{N}^{\mathrm{o}} 2$ la incidencia de mosca blanca $A$. floccosus en sus estados biológicos.

\begin{tabular}{|c|c|c|c|c|c|}
\hline \multirow{2}{*}{ Estados Biologicos } & \multicolumn{5}{|c|}{ Evaluación de mosca blanca en plantias en el tratamiento 2} \\
\hline & Hoja $N^{\circ} 1$ & Hoja $N^{\circ} 2$ & Hoja $N^{\circ} 3$ & Hoja $N^{\circ} 4$ & Promedio \\
\hline Adultos & 12 & 9 & 4 & 4 & 7,25 \\
\hline$N^{\circ}$ de Posturas & 7 & 12 & 8 & 6 & 8,25 \\
\hline Huevos & 16 & 14 & 10 & 8 & 12 \\
\hline Ninfas L1 & 13 & 9 & 9 & 7 & 9,5 \\
\hline Ninfas L2 & 13 & 8 & 9 & 8 & 9,5 \\
\hline Ninfas L3 & 0 & 0 & 9 & 9 & 4,5 \\
\hline Ninfas L4 & 0 & 0 & 10 & 9 & 4,75 \\
\hline Emisión Foliar & 0,78 & 0,78 & 0,78 & 0,78 & 0,78 \\
\hline Frecuencia & & & & & 7 Dias \\
\hline
\end{tabular}


Muestreo en el tratamiento 3

En el cuadro se detalla las poblaciones promedios, del tratamiento $\mathrm{N}^{\circ} 3$ la incidencia de mosca blanca $\boldsymbol{A}$. floccosus en sus estados biológicos.

\begin{tabular}{|c|c|c|c|c|c|}
\hline \multirow{2}{*}{ Estados Biologicos } & \multicolumn{5}{|c|}{ Evaluación de mosca blanca en plantias en el tratamiento 3} \\
\cline { 2 - 6 } & Hoja $\mathbf{N}^{\circ} \mathbf{1}$ & Hoja $\mathbf{N}^{\circ} \mathbf{2}$ & Hoja $\mathbf{N}^{\circ} \mathbf{3}$ & Hoja $\mathbf{N}^{\circ} \mathbf{4}$ & Promedio \\
\hline Adultos & 9 & 13 & 7 & 7 & 9 \\
\hline $\boldsymbol{N}^{\circ}$ de Posturas & 11 & 14 & 9 & 8 & 10,5 \\
\hline Huevos & 16 & 15 & 8 & 9 & 12 \\
\hline Ninfas L1 & 21 & 19 & 12 & 8 & 15 \\
\hline Ninfas L2 & 15 & 16 & 10 & 9 & 12,5 \\
\hline Ninfas L3 & 0 & 6 & 11 & 8 & 6,25 \\
\hline Ninfas L4 & 0 & 0 & 12 & 10 & 5,5 \\
\hline Emisión Foliar & 0,86 & 0,86 & 0,86 & 0,86 & 0,86 \\
\hline Frecuencia & & & & & 7 Dias \\
\hline
\end{tabular}

Muestreo en el tratamiento 4 testigo absoluto

En el cuadro se detalla las poblaciones promedios, del tratamiento $\mathrm{N}^{\circ} 4$ testigo absoluto, la incidencia de mosca blanca $A$. floccosus en sus estados biológicos.

\begin{tabular}{|c|c|c|c|c|c|}
\hline \multirow{2}{*}{ Estados Biologicos } & \multicolumn{5}{|c|}{ Evaluación de mosca blanca en plantias en el tratamiento 4} \\
\hline & Hoja N¹ & Hoja N² & Hoja N`3 & Hoja N4 & Promedio \\
\hline Adultos & 12 & 14 & 8 & 3 & 9,25 \\
\hline$N^{\circ}$ de Posturas & 10 & 12 & 6 & 7 & 8,75 \\
\hline Huevos & 19 & 16 & 12 & 9 & 14 \\
\hline Ninfas L1 & 24 & 17 & 15 & 11 & 16,75 \\
\hline Ninfas L2 & 18 & 8 & 7 & 6 & 9,75 \\
\hline Ninfas L3 & 0 & 0 & 12 & 10 & 5,5 \\
\hline Ninfas L4 & 0 & 0 & 11 & 9 & 5 \\
\hline Emisión Foliar & 0,80 & 0,80 & 0,80 & 0,80 & 0,80 \\
\hline Frecuencia & & & & & 7 Dias \\
\hline
\end{tabular}


Resultados del control biológico por medio de las liberaciones de ch. Carnea

En la presente tesis se estudió la aplicación en tres dosis en poblaciones de Ch. carnea, como controlador biológico en un numero de 100, 200, 300 larvas y en cada parcela experimental, comparando con un testigo absoluto al que no se realizó aplicación de ningún tipo, estos tratamiento fueron aplicados al área foliar de la planta con la finalidad de disminuir la Mosca blanca (A. floccosus).

Los resultados obtenidos en cada una de las parcelas, se diagnosticó mediantes sus estados ninfales de la mosca blanca A. floccosus L1, L2, L3, L4 y tomando en consideración el número de Ch. carnea que se liberó en cada uno de los tratamiento, durante las evaluaciones efectuadas en las semanas 23, 24, 27, 29, 32. Se obtuvo los siguientes resultados.

Resultados del muestreo en el estado ninfal de L 1

En los cuadro $\mathrm{N}^{\mathrm{o}} 8$ se detalla las evaluaciones realizadas desde la semana 23 a la 32 , en (T1 100 larvas, T2 200 larvas, T3 300 larvas, T4 testigo absoluto), fueron las siguientes.

Los valores promedio de los estado juvenil en L1, de la mosca blanca, se pudo observar que el promedio más bajo lo presento el tratamiento 3 (300 larvas de Ch. carnea) con 0,3 ninfas, seguido por el tratamiento 2 (200 larvas de Ch. carnea), con 1,4 ninfas, el tratamiento 1 (100 larvas de Ch. carnea), con 3 ninfas y el testigo con 12,8 ninfas de mosca blanca. 
Rafael V. Pluas-Pilozo; Alexandra A. Navarrete-Cornejo; Nuvia L. Morán-Sanchez

\begin{tabular}{|c|r|r|r|r|}
\hline \multirow{2}{*}{$\begin{array}{c}\text { REPETICIONES } \\
\text { (SEMANAS) }\end{array}$} & \multicolumn{5}{|c|}{ TRATAMIENTOS } \\
\cline { 2 - 5 } & $\begin{array}{c}100 \text { larvas } \\
(\mathrm{T} 1)\end{array}$ & $\begin{array}{c}200 \text { larvas } \\
(\mathrm{T} 2)\end{array}$ & $\begin{array}{c}300 \text { larvas } \\
(\mathrm{T} 3)\end{array}$ & $\begin{array}{c}\text { Testigo } \\
\text { absoluto } \\
\text { (T4) }\end{array}$ \\
\hline Semana 23 & 13,6 & 12,2 & 12,1 & 13,5 \\
\hline Semana 24 & 12,2 & 10,7 & 12,1 & 15,4 \\
\hline Semana 27 & 7,6 & 6,7 & 2,3 & 14,7 \\
\hline Semana 29 & 2,4 & 1,4 & 0,3 & 15,4 \\
\hline Semana 32 & 3 & 1,2 & 0,3 & 12,8 \\
\hline
\end{tabular}

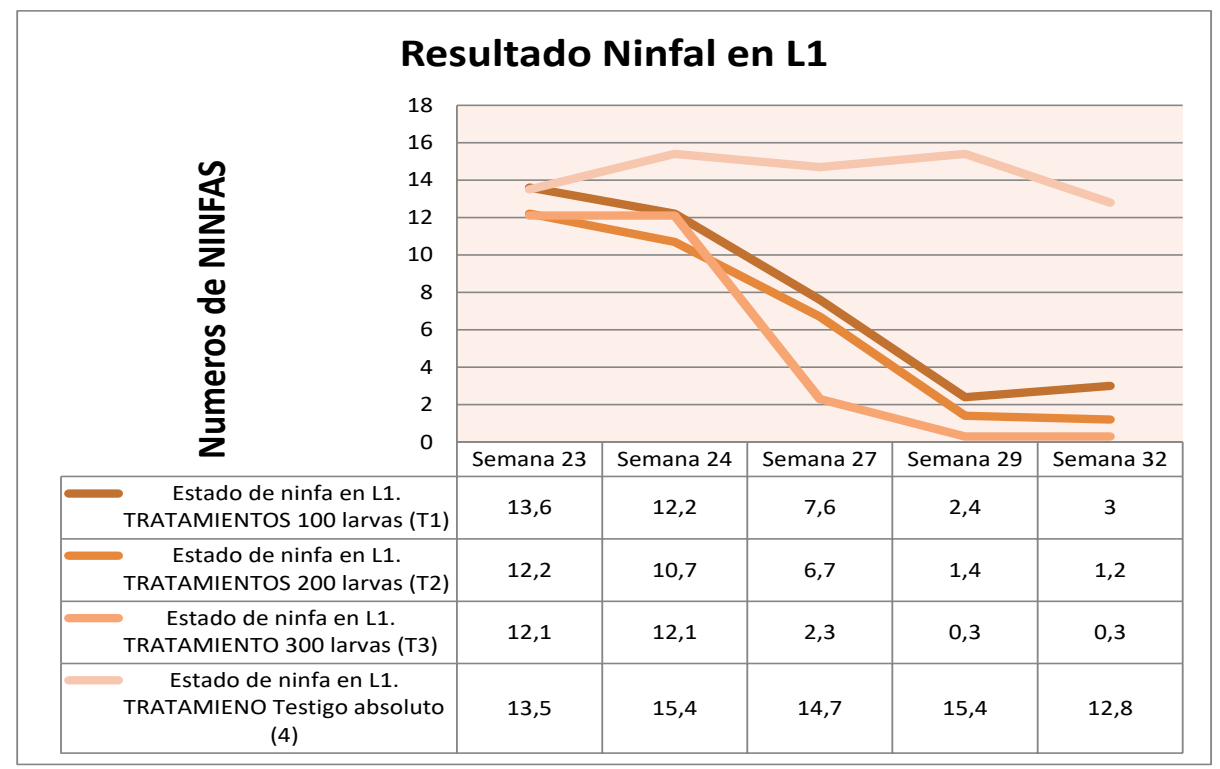

Resultados del muestreo en el estado ninfal de L 2

En cuadro de evaluación de L2, se observó, que el valor promedio más bajo lo presento el tratamiento 3 (300 larvas de $\boldsymbol{C h}$. carnea) con 0,5 ninfas, seguido por el tratamiento 2 (200 larvas de Ch. carnea), con 1,3 ninfas, el tratamiento 1 (100 larvas de $\boldsymbol{C h}$. carnea), con 3,7 ninfas, mostrando en el análisis estadístico, se considera una igualdad en los efectos de tratamientos entre sí, y en el testigo con 14,4 ninfas siendo altamente significativo para los demás tratamiento. 


\begin{tabular}{|c|r|r|r|r|}
\hline \multicolumn{5}{|c|}{ Estado de ninfa en L2 } \\
\hline $\begin{array}{c}\text { REPETICIONES } \\
\text { (SEMANAS) }\end{array}$ & $\begin{array}{c}\text { TRATAMIENTOS } \\
\text { (T1) larvas }\end{array}$ & $\begin{array}{c}\text { 200 larvas } \\
\text { (T2) }\end{array}$ & $\begin{array}{c}300 \text { larvas } \\
\text { (T3) }\end{array}$ & $\begin{array}{c}\text { Testigo } \\
\text { absoluto } \\
\text { (T4) }\end{array}$ \\
\hline Semana 23 & 11,2 & 11,4 & 12,4 & 12,6 \\
\hline Semana 24 & 10,8 & 11,4 & 11,2 & 15,1 \\
\hline Semana 27 & 9,3 & 4,9 & 2,2 & 14,7 \\
\hline Semana 29 & 4,3 & 2,1 & 0,5 & 14,5 \\
\hline Semana 32 & 3,7 & 1,3 & 0,5 & 14,4 \\
\hline
\end{tabular}

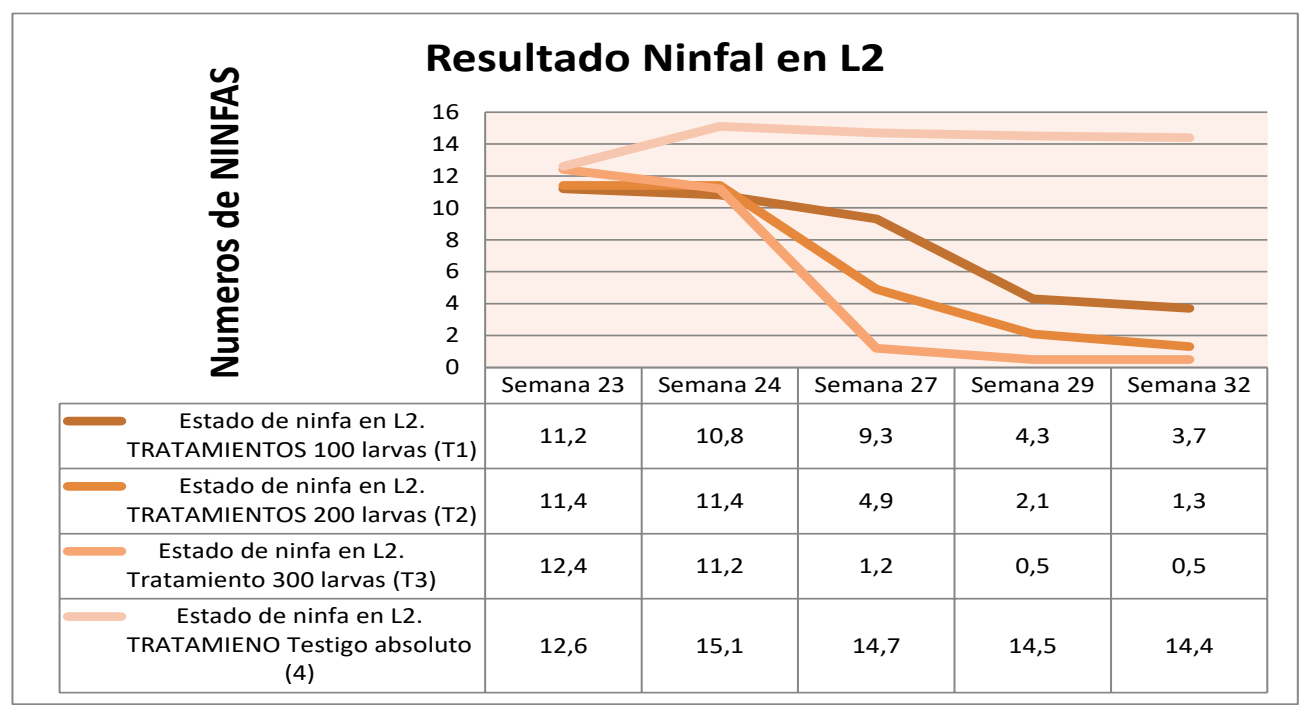

Resultados de la muestras en el estado ninfal de L 3

En el estado ninfal L3 se pudo observar que, el tratamiento 3 (300 larvas de Ch. carnea), presento el promedio más bajo con 0,8 ninfas, seguido por el tratamiento 2 (200 larvas de $\boldsymbol{C h}$. carnea) con 4,3 ninfas, y el tratamiento 1 (100 larvas de Ch. carnea), con 7,1 ninfas, y en el testigo un 20,7 ninfa siendo estadísticamente diferente entre sí. Véase en el cuadro 10. 
Rafael V. Pluas-Pilozo; Alexandra A. Navarrete-Cornejo; Nuvia L. Morán-Sanchez

\begin{tabular}{|l|r|r|r|r|}
\hline \multicolumn{5}{|c|}{ Estado de ninfa en L3 } \\
\hline $\begin{array}{c}\text { REPETICIONES } \\
\text { (SEMANAS) }\end{array}$ & $\begin{array}{c}\text { TRATAMIENTOS } \\
\text { 100 larvas } \\
\text { (T1) }\end{array}$ & $\begin{array}{c}\text { 200 larvas } \\
\text { (T2) }\end{array}$ & $\begin{array}{c}300 \text { larvas } \\
\text { (T3) }\end{array}$ & $\begin{array}{c}\text { Testigo } \\
\text { absoluto } \\
\text { (T4) }\end{array}$ \\
\hline Semana 23 & 0 & 0 & 0 & 0 \\
\hline Semana 24 & 8,2 & 7,9 & 8,2 & 8,7 \\
\hline Semana 27 & 8,3 & 5 & 2,4 & 14,2 \\
\hline Semana 29 & 7,1 & 4,5 & 1,6 & 23,1 \\
\hline Semana 32 & 7,1 & 4,3 & 0,8 & 20,7
\end{tabular}

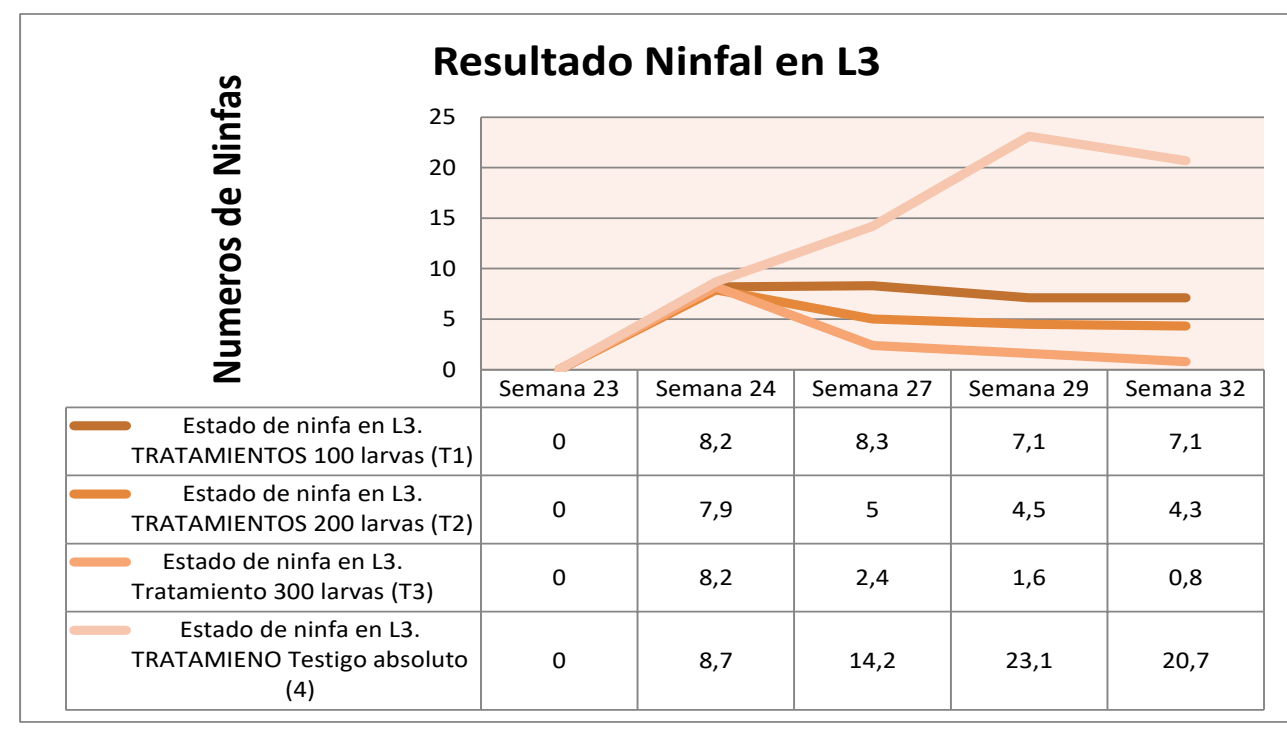

Resultados de la muestras en el estado ninfal de L 4

En el estado ninfal L4 se pudo observar que, el tratamiento 3 (300 larvas de Ch. carnea), presento el promedio con 2,9 ninfas, seguido por el tratamiento 2 (200 larvas de Ch. carnea) con 7,3 ninfas, y el tratamiento 1 (100 larvas de Ch. carnea), con 9,7 ninfas, y en el testigo un 23 ninfa, siendo estadísticamente diferente entre sí. Véase en el cuadro 11 


\begin{tabular}{|l|r|r|r|r|}
\hline \multicolumn{5}{|c|}{ Estado de ninfa en L4 } \\
\hline $\begin{array}{c}\text { REPETICIONES } \\
\text { (SEMANAS) }\end{array}$ & $\begin{array}{c}\text { TRATAMIENTOS } \\
\text { 100 larvas } \\
\text { (T1) }\end{array}$ & $\begin{array}{c}\text { 200 larvas } \\
\text { (T2) }\end{array}$ & $\begin{array}{c}300 \text { larvas } \\
\text { (T3) }\end{array}$ & $\begin{array}{c}\text { Testigo } \\
\text { absoluto } \\
\text { (T4) }\end{array}$ \\
\hline Semana 23 & 0 & 0 & 0 & 0 \\
\hline Semana 24 & 0 & 0 & 0 & 0 \\
\hline Semana 27 & 9,4 & 3,6 & 1,5 & 13,4 \\
\hline Semana 29 & 7,1 & 7,5 & 2,7 & 22,7 \\
\hline Semana 32 & 9,7 & 7,3 & 2,9 & 23
\end{tabular}

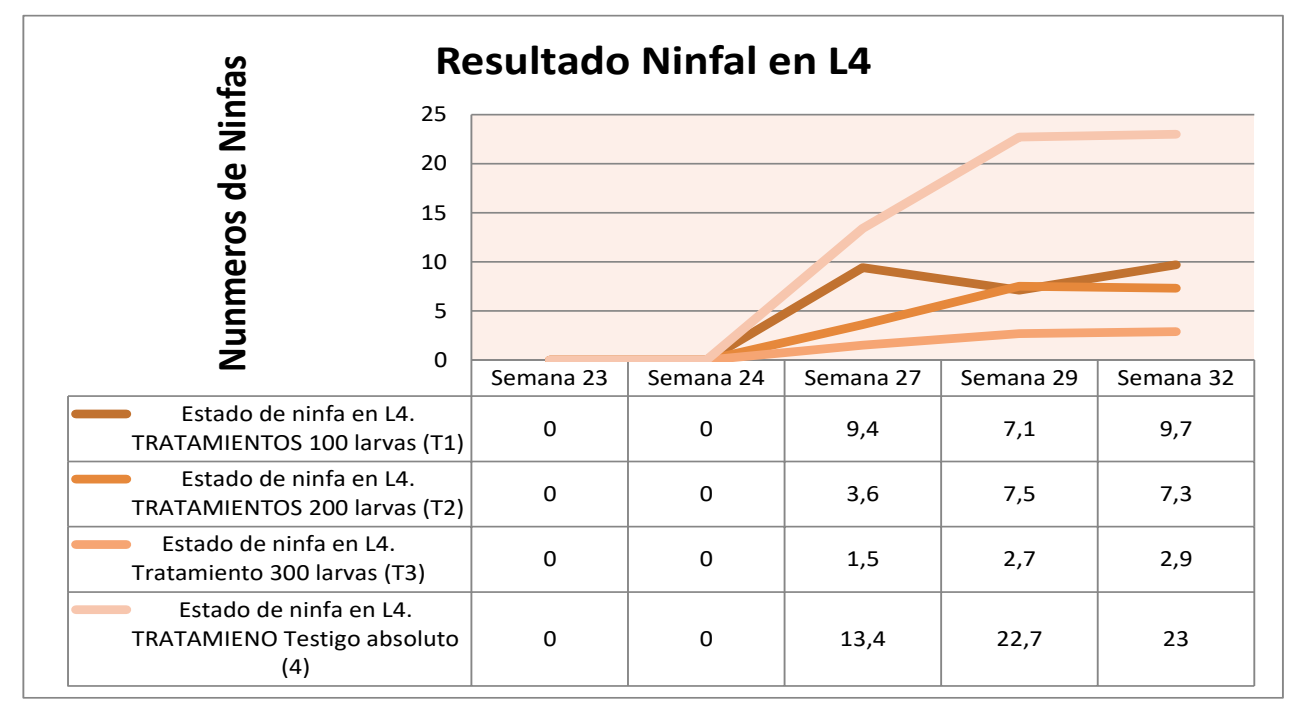

Análisis económico mediante el método de producción de ch. Carnea en laboratorio

El análisis económico mediante el método de producción podemos manifestar que la reproducción de 300 parejas de $\boldsymbol{C h}$. carnea, tiene un valor de 58.70 dólares, utilizando materiales de bajo costo, versus a la utilización de agroquímicos que existe en el mercado, teniendo un costo por hectárea de \$64,30 la atomización aérea, incluyendo el insecticida y aplicando de 4 a 6 ciclos al año en la época de mayor proliferación creando resistencia al insecto. 
Para el control del insecto -plaga se hacen liberaciones con un número de 400 a 500 huevos por hectárea, el millar de huevos tiene un costo de $\$ 10,60$, obteniendo un ahorro de $\$ 53.70$ en control, minimizando la contaminación ambiental.

A continuación se detalla en el siguiente cuadro $\mathrm{N}^{\circ} 8$, los materiales con los respectivos precios

\begin{tabular}{|c|c|c|c|c|}
\hline MATERIALES & UNIDAD & CANTIDAD & $\begin{array}{c}\text { PRECIO } \\
\text { UNITARIO }\end{array}$ & COSTO TOTAL \\
\hline Levadura de cerveza & Kg & 1 & 3,00 & 3,00 \\
\hline Algodón & Unidad & 1 & 1,00 & 1,00 \\
\hline Cartulina & Pliego & 1 & 0,70 & 0,70 \\
\hline Tijera & Unidad & 1 & 0,50 & 0,50 \\
\hline Pincel & Unidad & 1 & 0,50 & 0,50 \\
\hline Cilindro de carton & Unidad & 3 & 0,25 & 0,75 \\
\hline Huevos de Sitotroga & Kg & 1 & 2,00 & 2,00 \\
\hline Miel de abeja & Lt & 1 & 7,00 & 7,00 \\
\hline Malla metalica & Mt & 2 & 4,50 & 9,00 \\
\hline Cinta metrica & Unidad & 1 & 3,00 & 3,00 \\
\hline Tabla & Unidad & 1 & 7,00 & 7,00 \\
\hline Martillo & Unidad & 1 & 4,00 & 4,00 \\
\hline Clavos & Lb & 1 & 3,00 & 3,00 \\
\hline Bandejas Plasticas & Unidad & 3 & 0,75 & 2,25 \\
\hline Mano de obra & Jornal & Hora & 1,2 & 15,00 \\
\hline TOTAL & & & & 38,40 \\
\hline
\end{tabular}

Fuente: laboratorio de reproducción de Ch. carnea Hacienda Primobanano 2014. 


\section{Discusión.}

De acuerdo a los resultados de la investigación coincide con (Tigreros, 1989), (Arias \& Jinez, 1998) donde mencionan que Las primera fases de daños directos de Mosca blanca es en ninfa L1, L2, L3, L4, ya que en el presente trabajo se observó que en la fase L3 y L4 hubo mayor daño y presencia secreciones de mielecilla, por la extracción de savias de las hojas por los adultos y sus estados ninfales, siendo únicamente el estado de huevo y pupa los que no producen daños.

Analizando los comentarios de (Arias \& Jinez, 1998), donde menciona que la Mosca blanca en los daños indirectos son más importantes que los daños directos entre ellos se pueden mencionar el hongo saprofitos en especial "negrita o fumagina que puede llegar a cubrir toda la planta, dificultando la fotosíntesis, además puede producir el manchado de los frutos. En la presente investigación se pudo observar que si se impide sus fases de desarrollo, a los dos últimos estados ninfales no habrá daño del hongo fumagina por la poca presencia de la sustancia que segregan las ninfas.

En las practicas agronómicas mencionadas por (VIiñuela, 2000), como lucha biológica tiene una eficacia muy elevada mediante el uso de enemigos naturales principalmente parasitoides, depredadores y emtomapatógeno los cuales actúan en diferentes agro ecosistemas disminuyendo las poblaciones de estas plagas. De acuerdo con lo mencionado por (VIiñuela, 2000), si coincide con este trabajo investigativo, cuando un insecto es llevado de su medio natural a otro diferente de su población tiene un control muy satisfactorio y puede aumentar su reproducción rápidamente por la ausencia de sus enemigos naturales.

(VIiñuela, 2000) Nos menciona que el control de la mosca blanca ha dado en la mayoría de los casos resultados deficientes, debido sobre todo al elevado potencial reproductor y al solape entre 
generaciones y abundante secreción cerosa protege de manera muy eficiente al insecto. En lo cual no concuerda con lo obtenido en el presente trabajo ya que al emplear larvas de Ch. carnea en diferentes dosis se observó un buen control.

\section{Conclusiones.}

El trabajo de investigación presenta las siguientes conclusiones.

Establecido el número de Ch. carnea recopilado en campo, para iniciar la reproducción como pie de cría en el laboratorio, se logró la multiplicación de Ch. carnea relativamente en corto periodo (35 a 40 días) una población necesaria para las liberaciones del estudio de la investigación.

Los materiales para la reproducción Ch. carnea, son fácil de adquirir, y con un costo aceptable para su manejo.

En cuanto a la incidencia de la Mosca blanca A. floccosus, se pudo determinar al inicio de la evaluación (semana 23) una población promedio en los tratamiento 1 (13.6 ninfas) en 100 larvas de Ch. carnea , T2 (12,2 ninfas) en 200 larvas de Ch. carnea y T 3 (12,1 ninfas) en 300 larvas de Ch. carnea y a partir de la (semana 27) los niveles poblacionales se notaban diferentes, a las primera evaluación, en un promedio en el tratamiento 1 (7,6 ninfas), T2 (6,7 ninfas), T3 (2,3 ninfas) y en la semana 32 se pudo concluir que el tratamiento 3 (0.03 ninfas) se observó el mejor control liberando 300 larvas.

El análisis económico mediante la producción para las 300 parejas de Ch. carnea, tuvo un valor de \$ 58.70 para establecer el pie de cría, y tomando en cuenta el valor que se invierte por hectárea para el control de A. floccosus, es de $\$ 10.60$ liberando huevos de Ch. carnea y utilizando insecticidas tiene un costo de $\$ 64.30$ por hectárea, obteniendo un ahorro de $\$ 53.70$. Esto nos indica 
que es factible realizar este tipo de control por la inversión en el costo económico y ambiental al no utilizar agroquímicos que afectan al ecosistema.

\section{Bibliografía.}

Arias, M., \& Jinez, A. (1998). Biologia de Aleurothrixus floccosus Maskell u su relacion en la emision foliar del banano. Guayaquil: Acorbat 23.

Giffoni, J., Valera, N., Díaz, F., \& Vásquez, C. (2007). Ciclo biológico de Chrysoperla externa (hagen) (Neuroptera: Chrysopidae) alimentada con diferentes presas. Bioagro, 19(2).

Hoddel, M., Van Driesche, R., \& Sanderson, J. (1998). Biology and use of the whitefly parasitoid Encarsia formosa. Annual Review of Entomology, 43(1), 645-669.

Silvia N , L., Riquelme, M., \& Botto, E. (2010). Integración del control biológico y químico de la mosca blanca Trialeurodes vaporariorum (Hemiptera: Aleyrodidae). Revista Colombiana de Entomología, 36(2), 190-194.

Tigreros, J. (1989). Biologia y control de Mosca blanca de los Citricos. Aleurothirxus Floccosus Maskell ( Homoptera: Alerodidae). En D. g. Zusammenarbeir, Sanidad vegetal del Ministerio de Agricultura y Ganaderia (pág. 40 53). India.

VIiñuela, E. (2000). La resistencia de insecticidas y plagas orticulas en españa. Almeria: sociacion AGROUniversidad de Alemania. 